\title{
The Clinical Impact and Cost Effectiveness of Quadrivalent Versus Trivalent Influenza Vaccination in Finland
}

\author{
Lisa Nagy $^{1} \cdot$ Terho Heikkinen $^{2} \cdot$ Alfred Sackeyfio $^{3} \cdot$ Richard Pitman $^{1}$
}

Published online: 16 July 2016

(c) The Author(s) 2016. This article is published with open access at Springerlink.com

\begin{abstract}
Background Trivalent influenza vaccines encompass one influenza B lineage; however, predictions have been unreliable on which of two antigenically distinct circulating lineages will dominate. Quadrivalent seasonal influenza vaccines contain strains from both lineages. This analysis assesses the cost effectiveness of switching from trivalent inactivated influenza vaccination (TIV) in Finland to quadrivalent vaccination, using inactivated (QIV) or liveattenuated (Q-LAIV) vaccines.

Methods A transmission model simulated the dynamics of influenza infection while accounting for indirect (herd) protection. Prior distributions for key transmission parameters were repeatedly sampled and simulations that fitted the available information on influenza in Finland were recorded. The resulting posterior parameter distributions were used in a probabilistic sensitivity analysis in which economic parameters were sampled, simultaneously encompassing uncertainty in the transmission and economic parameters. The cost effectiveness of a range of
\end{abstract}

Electronic supplementary material The online version of this article (doi:10.1007/s40273-016-0430-z) contains supplementary material, which is available to authorized users.

Lisa Nagy

richard.pitman@iconplc.com

1 ICON Health Economics and Epidemiology, 100 Park Drive, Milton Park, Oxfordshire, OX14 4RY, Oxford, UK

2 Department of Pediatrics, University of Turku and Turku University Hospital, Turku, Finland

3 AstraZeneca, Cambridge, UK trivalent and quadrivalent vaccine policies over a 20 -year time horizon was assessed from both a societal and payer perspective in 2014 Euros.

Results The simulated temporal incidence pattern of symptomatic infections corresponded well with case surveillance data. A switch from the current TIV to Q-LAIV in children ( 2 to $<18$ years) and to QIV in other ages was estimated to annually avert approximately 76,100 symptomatic infections (95\% range 36,700-146,700), 11,500 primary care consultations $(6100-20,000), 540$ hospitalisations (240-1180), and 72 deaths (32-160), and was cost-saving relative to TIV ( $€ 374$ million averted [€161-€752], in 2014 Euros, discounted at $3 \%$ ). This scenario had the highest probability of being the most costeffective scenario considered.

Conclusions This analysis demonstrates that quadrivalent vaccination is expected to be highly cost effective, reducing the burden of influenza-related disease.

\section{Key Points}

Dynamic transmission model incorporating indirect (herd) protection.

Probabilistic model, calibrated to data, simultaneously accounts for uncertainty in transmission, clinical and economic parameters.

Costs and quality-adjusted life-years of various influenza vaccination scenarios in Finland were compared.

A quadrivalent scenario, with live-attenuated vaccine in children 2 to $<18$ years of age, was the scenario with the highest expected net benefit. 


\section{Introduction}

The significant annual health and economic burden of influenza has prompted a number of studies to assess potentially more effective and cost-effective ways of controlling this infection [1-5].

Evidence is accumulating that paediatric influenza vaccination is an efficient and cost-effective approach to alleviating the burden of influenza in children, as well as in the wider population $[2,3]$. Finland was one of the first countries to implement routine paediatric influenza vaccination [6] and currently recommends immunising all children between the ages of 6 and 35 months, in addition to seniors 65 years or older and persons of any age over 36 months at increased risk of influenza-related complications.

The co-circulation, since 2002 [7], of two genetic lineages of influenza B (Yamagata and Victoria) has prompted a move away from the use of trivalent vaccines, containing antigens to two influenza A strains (one from each of the currently circulating subtypes, AH1N1 and AH3N2) and one influenza B lineage strain, to a vaccine containing antigens to all four strains. These quadrivalent vaccines avoid the need to predict which $B$ lineage will circulate in the following influenza season, a prediction that has been correct in only four of the ten seasons after Victoria first circulated in Finland. In three other seasons, either both influenza B strains circulated or the selected strain showed only sporadic incidence. In three seasons, the wrong strain was selected [7-9]. This situation is not unique to Finland, with mismatch years occurring wherever trivalent vaccines are used $[4,10]$.

Several studies looking to estimate the public health and economic impact of switching from a trivalent to a quadrivalent seasonal influenza vaccine have used a static modelling approach with retrospective data to study health outcomes $[11,12]$. Static models are unable to account for dynamic changes in the probability of infection and hence these studies failed to account for indirect protection and, as a result, may underestimate the impact of vaccination. Current recommended practice [13] is to use a dynamic model when evaluating infectious disease interventions that are likely to have an impact on transmission. Few other published studies to date have taken transmission dynamics into account when comparing the effects of trivalent and quadrivalent vaccination on seasonal influenza $[5,14,15]$.

This paper uses a dynamic transmission model to examine the efficacy and cost-utility of nationally replacing currently used trivalent influenza vaccines in Finland with quadrivalent vaccines, either inactivated or live-attenuated. This model uses a probabilistic sensitivity analysis (PSA) approach to take into account the uncertainty in the transmission, clinical and economic parameters.

\section{Methods}

A compartmental transmission model was used to simulate the population-level dynamics of influenza infection (Sect. 2.1). A set of key transmission parameters was repeatedly sampled from input distributions and the transmission model was numerically integrated; those sets whose results fit the available information on influenza in Finland were retained, thus calibrating the model (Sect. 2.2). A set of updated transmission parameter distributions was thus obtained, and the transmission model was integrated with these parameter sets, to produce a PSA of the transmission model inputs. The results of integrating the model with these parameters were used as inputs to an economic PSA in which the clinical and economic parameters were sampled (Sect. 2.3). The model therefore simultaneously encompassed uncertainty in the transmission parameters in addition to the economic inputs.

\subsection{Dynamic Transmission Model}

The dynamic transmission model had an SEIRS(V) structure [16] (Susceptible, Exposed, Infectious, Recovered, Vaccinated), stratified by age in months (see the electronic supplementary material, which provides details and equations). Ageing was simulated on a monthly basis, with population [17] and lifetable [18] data informing the demographics. Individuals within an age group were assumed to mix at random. Estimates of the relative rate of contact between individuals in the same and in disparate age groups were derived from the POLYMOD study [19] and recorded in a contact matrix. The product of these agespecific contact rates and the per contact probability of transmission yielded an age-stratified matrix of transmission coefficients. The magnitude of these transmission coefficients was assumed to vary sinusoidally over time to reflect the observed seasonality in influenza transmission. Imported influenza infections were seeded into the model each autumn. Vaccination was assumed to confer immediate protection and to have been completed prior to the start of the influenza season, being applied on 1 September each year.

Four influenza viruses were assumed to circulate: AH1N1, AH3N2, B Yamagata, and B Victoria; the model assumed no cross-protection between these viruses. Within each subtype or lineage, influenza strains were not distinguished. The waning of immunity over time, whether naturally-acquired or vaccine-induced, simulated a combination of antigenic drift and the waning of immunity with age. The annual vaccine strain composition was obtained from World Health Organization (WHO) recommendations [20]. 
The pre-existing immunity structure (proportion of the population immune, by age and virus) at the start of the model was estimated by running the model forward, using previously published parameter inputs [21] adapted for Finland's population size, to estimate the susceptible population by age at the start of an influenza season (see the electronic supplementary methods for details).

\subsection{Model Calibration}

The model was calibrated to observed data on the incidence of symptomatic influenza infection in children [22]. Resulting parameter combinations were thus consistent with this observed epidemiology. In the calibration, the transmission inputs underwent Monte Carlo sampling followed by numerical integration of the model from the $2000 / 2001$ to $2008 / 2009$ seasons. The resulting simulated influenza incidence was compared with epidemiological data from the same period, using a set of defined fit criteria. To do so, the incidence of infection was converted to that of symptomatic infections by applying the conditional probability of influenza symptoms, given that infection had occurred (described in the electronic supplementary methods).

\subsubsection{Data}

Data used to calibrate the model were estimates of the incidence of symptomatic influenza infection in children from Turku, Finland, over the 2000/2001 and 2001/2002 seasons [22]. WHO [23] surveillance reports provided an indication of the pre-pandemic temporal variation in influenza incidence.

Finnish National Institute for Health and Welfare (THL) [8] surveillance reports were used as an independent check of the temporal pattern of variation in incidence in the calibrated model; in order to compare the variation in incidence published in these reports with that simulated by the model, on the same scale, the two datasets were each normalised by dividing through by their respective overall mean incidence.

These data and the process of normalisation are described in more detail in the electronic supplementary methods.

\subsubsection{Probabilistic Sampling}

Key inputs are summarized in Table 1 . Sets of transmission parameters were drawn from assigned input distributions. If the corresponding simulated incidence of symptomatic infection, for each virus separately, met the following heuristic criteria, the simulation was retained:

I. (a) AH1N1, AH3N2, and B Yamagata: Fell within a predefined deviance of the Turku data [22] (20 for H1N1 and Yamagata, 150 for H3N2). Deviance was calculated as the sum of the individual Poission deviances between model and data for each age group and each season over 2000/2001 to 2001/2002 (see the electronic supplementary methods for further details).

(b) B Victoria: When introduced in 2002/2003, the cumulative seasonal incidence in that season fell within a range consistent with seasonal influenza [7].

II. Ratio of standard deviation/mean cumulative seasonal incidence for each virus within the range of the same measure as derived from WHO surveillance data [23].

III. A low incidence prior to the usual start of the influenza season in week 40 (less than approximately $1 \%$ of the population).

The B Yamagata lineage was calibrated first, with all transmission inputs sampled. Parameters were assumed to be common across influenza strains unless there was sufficient information to assume otherwise. Common parameters were not resampled in the subsequent calibration of the other strains. A lack of detailed data for the Victoria lineage meant that all parameters were set equal to those of the corresponding Yamagata simulation, except for vaccine efficacy and a virus-specific scale factor applied to the pre-

Table 1 Key transmission model inputs

\begin{tabular}{|c|c|c|c|c|}
\hline Input & Minimum & Maximum & Sampling distribution & Stratified by \\
\hline \multicolumn{5}{|l|}{$R_{0}$ parameters } \\
\hline Transmission coefficient & $1.25 \mathrm{E}-07$ & $3.75 \mathrm{E}-07$ & Uniform & Virus $^{\mathrm{a}}$ \\
\hline Latent period (days) & 0.01 & 3 & Uniform & Virus $^{\mathrm{a}}$ \\
\hline Infectious period (days) & 0.5 & 5 & Uniform & Virus $^{\mathrm{a}}$ \\
\hline \multicolumn{5}{|l|}{ Immunity parameters } \\
\hline Duration of naturally-acquired immunity (years) & 0.5 & 75 & Uniform & Virus $^{\mathrm{a}}$ \\
\hline Duration of vaccine-induced immunity (years) & 0.5 & 3 & Uniform & Virus $^{\mathrm{a}}$ \\
\hline Pre-existing immunity & \multicolumn{3}{|c|}{ See Fig. S1.2 in the electronic supplementary methods } & Virus \\
\hline
\end{tabular}

${ }^{\mathrm{a}} \mathrm{B}$ Victoria inputs were set equal to B Yamagata samples for each simulation 
existing immunity age profile. For each influenza A subtype, efficacy and the pre-existing immunity scale factor were also resampled, as well as the transmission coefficient, infectious period, latent period, and durations of immunity (naturally-acquired and vaccine-induced). A detailed list of these inputs and their distributions is provided in the electronic supplementary methods.

The model was calibrated over the first two seasons (2000/2001 to 2001/2002), and each set of parameter samples whose results met the heuristic criteria above was retained; the group of all these sets of parameter samples was collectively referred to as the calibrated model.

The transmission model simulated the dynamics of influenza infection, irrespective of clinical presentation (symptomatic or asymptomatic). During calibration, the probability of symptoms, given influenza infection, was also sampled for each run in order to calibrate the cumulative seasonal incidence of each strain to the symptomatic infection data [22] for that season. All four viruses were assumed to be equally likely to produce symptoms.

For each parameter set in the calibrated model, the model was then run from $2000 / 2001$ to $2008 / 2009$. The resulting cumulative seasonal incidences were aggregated over all four strains to yield the seasonal incidence of total symptomatic influenza, and subsequently averaged over all seasons and all simulations to produce the expected (mean) seasonal incidence for use in the following risk function calculations.

\subsubsection{Clinical Outcomes and Risk Functions}

Age-stratified risk functions translated symptomatic infections into primary care physician consultations, hospitalisations, and deaths.

Finnish estimates were obtained for the mean annual number of influenza-attributable hospitalisations in children [24] and deaths in all ages [25]. These age-stratified estimates were divided by the age-stratified expected seasonal incidence of symptomatic infections to obtain the age-stratified probability of an outcome, given the influenza symptoms. These probabilities were not stratified by influenza strain due to a lack of available data to indicate variation by strain.

Age-stratified risk functions (probabilities of an outcome, given the symptoms) for hospitalisations in adults, and for general practitioner (GP) consultations in all ages, were based on estimates from a previous adaptation of this model for the UK $[21,26]$.

\subsection{Expected Net Benefit Analysis}

In this analysis, risk functions and economic estimates were applied to the calibrated model output to calculate clinical and economic outcomes, which were then compared for six vaccination scenarios described below.

Each parameter set obtained by the calibration was reused once and the model was again integrated from the 2000/2001 season, and then projected past 2014 over a 20 -year time horizon. The risk functions and clinical and economic parameters were then sampled in a Monte Carlo simulation and applied to the transmission results.

\subsubsection{Vaccination}

Key vaccination inputs are reported in Table 2 and Fig. 1.

2.3.1.1 Scenarios For each set of transmission parameter samples obtained during the calibration process, six vaccination scenarios were run (Table 3 ). These were chosen in order to explore the cost effectiveness of switching from the currently used trivalent inactivated influenza vaccine (TIV) to the quadrivalent equivalent (QIV), and then the potential cost effectiveness of replacing QIV with a quadrivalent live-attenuated influenza vaccine (Q-LAIV) in those age groups in which it is licensed (children aged 2 to $<18$ years). Complete replacement of QIV with Q-LAIV over this age range was analysed, in addition to an equal market split between QIV and Q-LAIV in these children. In order to be able to quantify the value of current treatment, a scenario without vaccination was also included.

These scenarios followed the status quo until diverging in the 2014/2015 season, with the exception of the scenario without vaccination, in which there was no vaccination from the 2000/2001 season onwards. The strain composition of trivalent vaccines was obtained from WHO recommendations [20] up to and including the 2013/2014 season. In scenarios with quadrivalent vaccination, all relevant vaccines were assumed to

Table 2 Key vaccination inputs

\begin{tabular}{llllll}
\hline Age group (years) & \multicolumn{2}{l}{ Inactivated } & & \multicolumn{2}{l}{ Live } \\
& Mean & $95 \% \mathrm{CI}$ & & Mean & $95 \% \mathrm{CI}$ \\
\hline Vaccine efficacy & & & & \\
0 to $<18[36]$ & 48 & $31-61$ & & 80 & $70-87$ \\
18 to $<65[36]$ & 59 & $50-66$ & & $39^{\mathrm{a}}$ & $16-55$ \\
$65+[47]$ & 50 & $39-59$ & & - & - \\
Vaccine dosage costs & & & & \\
0 to $<65$ & 7.18 & - & & $25.75^{\mathrm{a}}$ & - \\
$65+$ & 7.05 & - & - & - \\
\hline
\end{tabular}

CI confidence interval, PSA probabilistic sensitivity analysis

${ }^{a}$ Live-attenuated vaccine had $0 \%$ market share in those aged $\geq 18$ years in all modelled scenarios

b Dosage costs were held fixed in the PSA; assumptions were considered in sensitivity analyses (Sect. 2.3.3) 


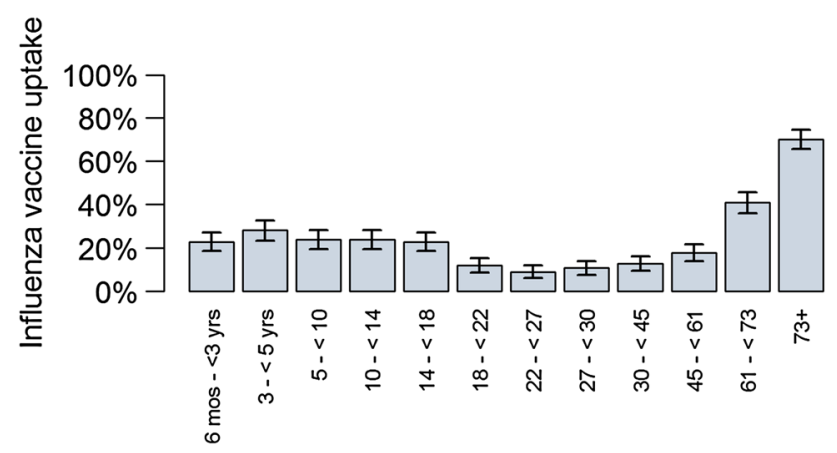

Fig. 1 Vaccine uptake, from a TNS Gallup Oy survey, sampled in the probabilistic sensitivity analysis

entirely switch to quadrivalent during 2014. Trivalent scenarios followed a random pattern, consistent with the observed $50 \%$ probability of a trivalent vaccine match to the circulating strain of influenza B, from the 2014/2015 season onwards to determine which influenza B lineage was included in the vaccine. For scenarios in which liveattenuated vaccine was modelled in children, the switch was also assumed to occur entirely during the 2014/15 season.

The 50 and $100 \%$ in some scenario names refer to the proportion of administered vaccines that were live-attenuated, and have no relation to the influenza vaccine uptake in the scenario.

2.3.1.2 Uptake Where possible, vaccine uptake prior to the 2012/13 season was based on data, with any unknown quantities over this period sampled in the calibration. An online survey was carried out from a nationally representative register of respondents, based on members of the general public, to estimate the vaccination uptake in Finland, by age, in the 2012/2013 season (Fig. 1). The resulting estimates were applied in all age groups from 2012/2013 onwards, sampled within confidence limits. For a given simulation, all vaccination scenarios used the same sampled uptake value.
Details of both the survey and the probabilistic sampling of both the initial vaccination and the survey estimates are described in the electronic supplementary methods.

\subsubsection{Clinical and Economic Outcomes}

For each model simulation, the cumulative age-stratified numbers of symptomatic infections were recorded from mid-2014 to mid-2034, both undiscounted and discounted at $3 \%$ [27] from the start of the 2014/2015 influenza season. The risk functions were applied to these cumulative estimates to obtain clinical outcomes; estimated costs and quality-adjusted life-years (QALYs) lost were then applied to these outcomes.

Price parity between trivalent and quadrivalent vaccines (TIV $=$ QIV and LAIV $=$ Q-LAIV) was assumed. Costs were split into direct medical costs, direct travel costs, and indirect productivity costs, which included the opportunity costs of absenteeism, caring for an ill child, time to attend a vaccination appointment, and the (human capital) productivity costs foregone due to premature death. Costs were obtained from national reports [28], price lists [29], and previous cost-effectiveness analyses [3], inflated to 2014 values (first quarter) using price and salary indices [30, 31]. Productivity costs were calculated, and costs are presented from both a societal and payer perspective [32].

A QALY loss due to morbidity [33] was applied to each modelled symptomatic infection, and age-specific QALYs [34] lost were applied to each influenza-attributable premature death [34].

The base-case analysis was obtained by averaging the clinical and economic outputs over all simulations. The ranges in which $95 \%$ of simulation outputs fell were also calculated for each outcome.

A set of cost-effectiveness acceptability curves was calculated to illustrate the probability of each scenario being cost effective over a range of cost-effectiveness thresholds. As the objective of most health policies is to maximise health, a cost-effectiveness acceptability frontier was also derived, which illustrates the probability that the

Table 3 Vaccination scenarios

\begin{tabular}{ll}
\hline Scenario name & Description \\
\hline No vaccination & No vaccination in any age, from $2000 / 2001$ and for the entire model run \\
TIV & Status quo: TIV in individuals 6 months of age and over \\
QIV & QIV, 6 months and older \\
TIV/Q-LAIV $(100 \%)$ & TIV, 6 to $<24$ months and $18+$ years; Q-LAIV, 2 to $<18$ years $(100 \%$ market share $)$ \\
QIV/Q-LAIV $(50 \%)$ & QIV, 6 to $<24$ months and $18+$ years; Q-LAIV and QIV, 2 to $<18$ years $(50 \%$ market share each) \\
QIV/Q-LAIV $(100 \%)$ & QIV, 6 to $<24$ months and $18+$ years; Q-LAIV, 2 to $<18$ years $(100 \%$ market share $)$
\end{tabular}

$T I V$ trivalent inactivated vaccine, $Q I V$ quadrivalent inactivated vaccine, $Q$ - $L A I V$ quadrivalent live-attenuated influenza vaccine 
scenario with the highest expected net health benefit (ENHB) is cost effective [35].

\subsubsection{Sensitivity Analyses}

A univariate sensitivity analysis was run to explore the sensitivity of the model results to an increase or decrease of $10 \%$ in key parameter values. The overall mixing matrix structure could not be altered in the univariate analysis, therefore a sensitivity analysis was also run directly on this structure. Dosage cost assumptions (price parity and list prices) were tested, and a break-even analysis compared dosage prices for select scenarios. The structural assumption of density- versus frequency-dependent forces of infection was considered by comparing both methods (see the electronic supplementary methods and results for details).

In its entirety, the model was run as a PSA on the transmission, clinical and economic parameters. This approach bolstered the robustness of the results and decreased the need, seen in prior models [21], for scenario or extreme value analyses to help quantify the uncertainty in the transmission model results.

\section{Results}

\subsection{Calibration}

Of approximately 13,000,000 parameter samples, 8998 simulations were amassed that fit the heuristic criteria (I)(III).

An encouragingly close correspondence was achieved between the normalised monthly incidence of symptomatic infection output by the model and the normalised THL case surveillance data [8] (Fig. 2). Although the model was only calibrated to (non-THL) data [22] from the 2000/2001 and 2001/2002 seasons, this figure shows that the observed incidence of infection was similar to the average model incidence of infection, and was encompassed by the upper limit of the $95 \%$ range of the calibrated model in nearly all pre-pandemic seasons. Direct comparison between the model average and the THL data must be undertaken with caution as the average over all calibrated model runs will show far less variation than the results of individual parameter sets in the calibrated model. Additionally, the THL surveillance data are themselves an estimate of the true incidence. The relative incidence of influenza A and B is also similar between the THL data and the model outcomes, even though this ratio was not one of the model fit heuristics. The correspondence is encouraging as these THL data were only used for validation and not for the calibration of the model.
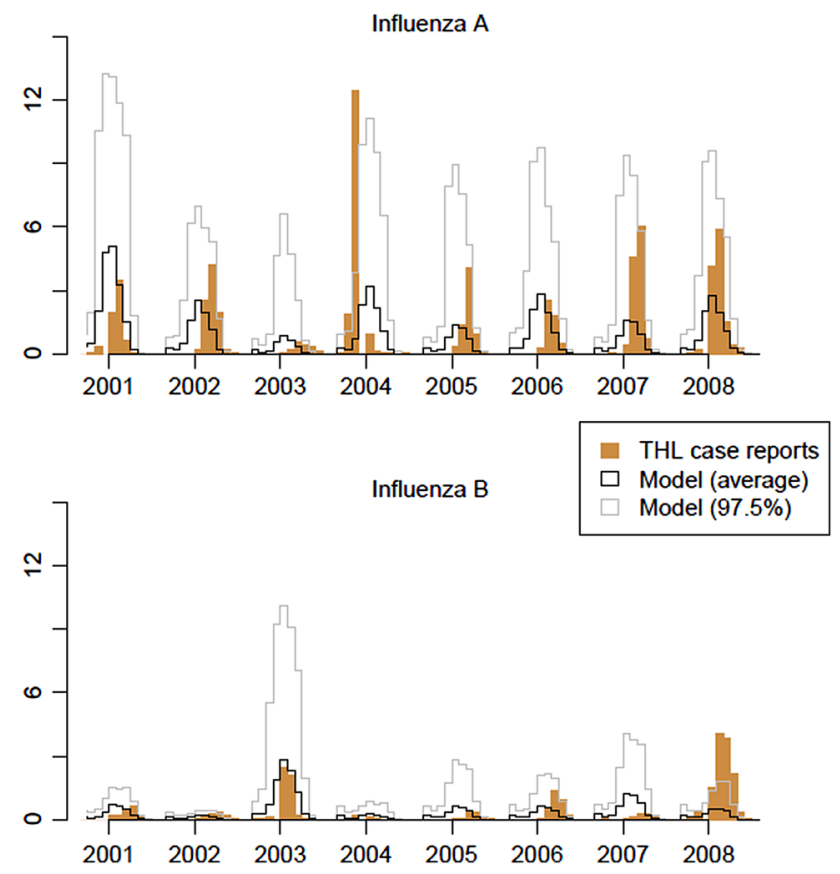

Fig. 2 Monthly incidence of symptomatic influenza, averaged over all runs and normalised to the 2000/2001 to 2007/2008 overall average. Comparison of the normalised modelled monthly incidence of symptomatic influenza infection to normalised National Institute for Health and Welfare (THL) surveillance reports [8] of symptomatic influenza. Figure derived from THL data, with permission

Sampling from uniform input distributions, and keeping the samples that met the calibration criteria, produced clearly defined unimodal distributions (Fig. 3) for the basic reproduction number (the number of secondary infections produced by an average primary infection in a totally susceptible population, $R_{0}$ ); these distributions were clearly updated from their initial inputs by the acceptance-rejection sampling according to the calibration heuristic (Fig. 4). Other updated parameter distributions are reported in the electronic supplementary results.

\subsection{Clinical Outcomes}

The undiscounted clinical outcomes predicted by the model were averaged over all simulations to give the expected average annual clinical burden of influenza in Finland over the 20-year time horizon of the model; these outcomes are summarised in Table 4. The expected values are presented, along with ranges, which represent the intervals in which $95 \%$ of the 8998 simulations fell.

On average, a switch from the current trivalent inactivated vaccines to QIVs was estimated to annually avert 40,500 symptomatic infections $(95 \%$ range $15,700-98,400), 6100$ primary care physician consultations (2700-13,300), 360 hospitalisations (140-940) and 54 deaths (22-135). 

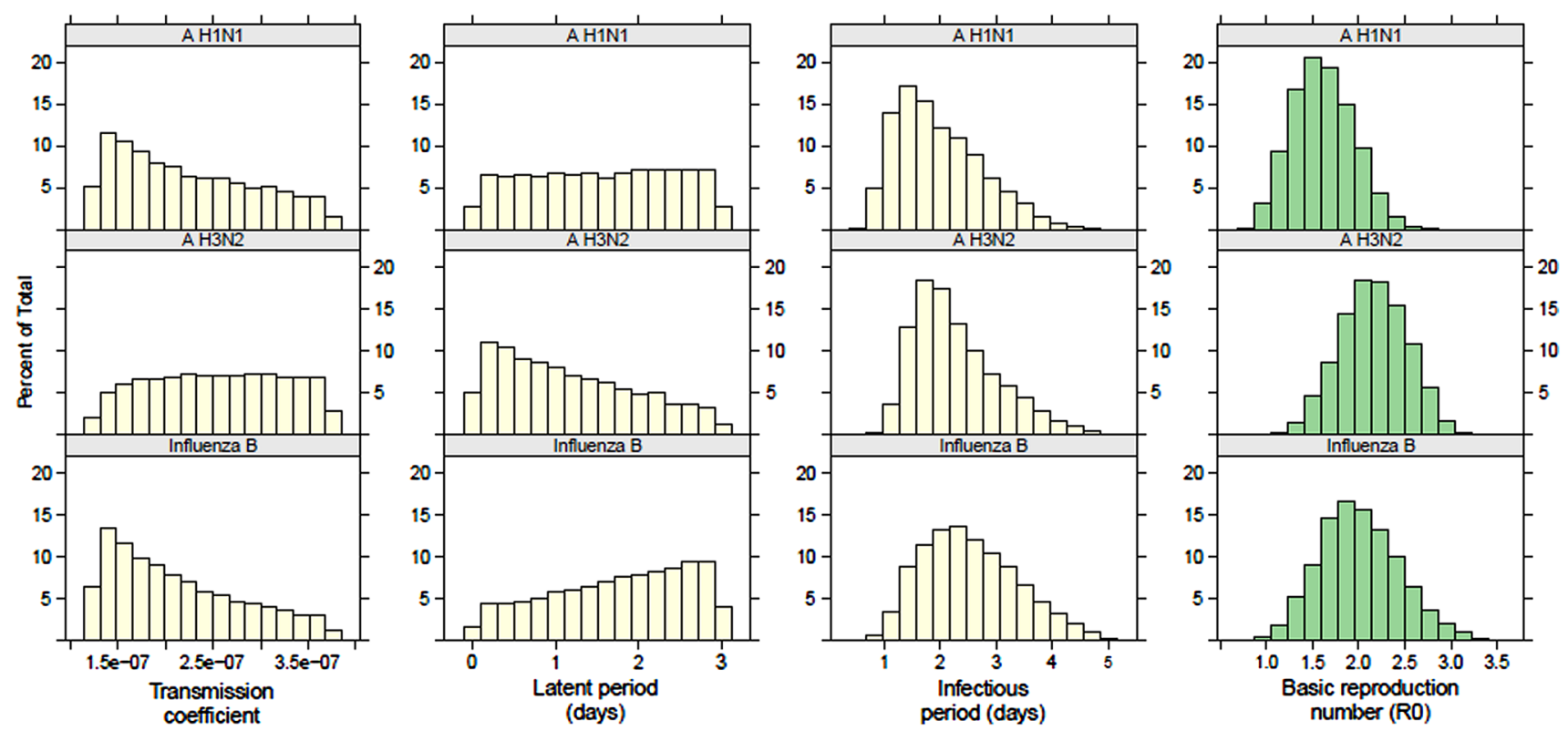

Fig. 3 Distribution of parameter samples over 8998 runs: calibrated distributions, by virus, of selected parameters contributing to the basic reproduction number $\left(R_{0}\right)$ and resultant $R_{0}$ distributions

Fig. 4 Distribution of basic reproduction number $\left(R_{0}\right)$ parameter samples over 8998 runs: comparison between initial input distributions and updated (calibrated) distributions, by virus
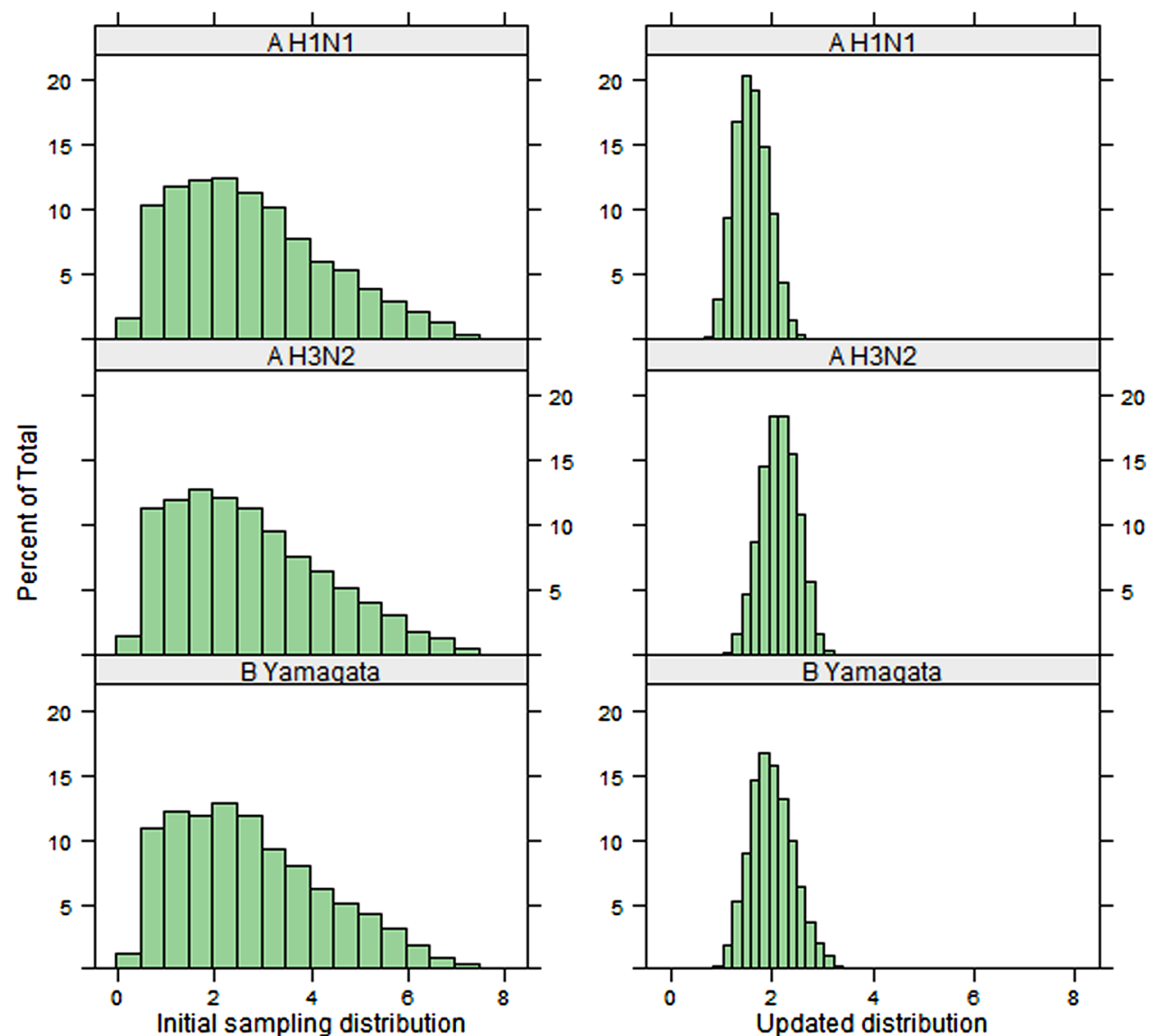

Due to the higher efficacy, by strain, of live-attenuated vaccination in children [36], using exclusively Q-LAIV in children 2 to $<18$ years of age rather than exclusively using QIV in everyone aged 6 months and over was estimated to avert, on average, an additional 35,600 symptomatic infections $(16,400-64,000), 5300$ primary care 
Table 4 Undiscounted 20-year average annual number of clinical outcomes in Finland

\begin{tabular}{|c|c|c|c|c|c|c|c|c|}
\hline \multirow[t]{2}{*}{ Scenario } & \multicolumn{2}{|l|}{ Symptomatic infections } & \multicolumn{2}{|l|}{ GP consultations } & \multicolumn{2}{|l|}{ Hospitalisations } & \multicolumn{2}{|l|}{ Deaths } \\
\hline & Exp (range) & Rate $^{\mathrm{a}}$ & Exp (range) & Rate $^{\mathrm{a}}$ & Exp (range) & Rate $^{\mathrm{a}}$ & Exp (range) & Rate $^{\mathrm{a}}$ \\
\hline No vaccination & $461,658(251,158-796,580)$ & 8724 & $62,139(35,731-104,489)$ & 1174 & 3758 (1553-7374) & 71 & $516(213-997)$ & 9.7 \\
\hline TIV & $357,454(205,615-581,248)$ & 6755 & $46,839(27,820-74,934)$ & 885 & $2276(988-4459)$ & 43 & $292(126-559)$ & 5.5 \\
\hline QIV & $316,988(170,321-531,924)$ & 5990 & $40,702(22,538-67,269)$ & 769 & $1913(745-3897)$ & 36 & $238(90-476)$ & 4.5 \\
\hline $\begin{array}{l}\text { TIV/Q-LAIV } \\
100 \%\end{array}$ & $302,515(152,172-516,418)$ & 5716 & $38,193(19,608-65,024)$ & 722 & $1972(732-4066)$ & 37 & $257(95-517)$ & 4.8 \\
\hline $\begin{array}{l}\text { QIV/Q-LAIV } \\
50 \%\end{array}$ & $298,779(151,774-511,376)$ & 5646 & $37,948(19,856-63,905)$ & 717 & $1826(668-3788)$ & 34 & $229(82-464)$ & 4.3 \\
\hline $\begin{array}{l}\text { QIV/Q-LAIV } \\
100 \%\end{array}$ & $281,389(135,239-490,111)$ & 5317 & $35,361(17,372-60,708)$ & 668 & $1741(600-3677)$ & 33 & $220(75-455)$ & 4.2 \\
\hline
\end{tabular}

Scenario names are defined in Table 3

$G P$ general practitioner, $T I V$ trivalent inactivated vaccine, $Q I V$ quadrivalent inactivated vaccine, $Q$ - $L A I V$ quadrivalent live-attenuated influenza vaccine, Exp expectation (average) over all simulations, range range in which $95 \%$ of simulations lay

${ }^{\text {a }}$ Rate/100,000 person years

Table 5 Cumulative discounted costs $(2014 €)$, QALY losses and incremental cost-effectiveness ratios of scenarios

\begin{tabular}{|c|c|c|c|c|c|}
\hline Scenario & $\begin{array}{l}\text { Total costs } \\
(€, \text { millions })\end{array}$ & $\begin{array}{l}\text { Total QALYs } \\
\text { lost (thousands) }\end{array}$ & $\begin{array}{l}\Delta \text { Costs } \\
(€, \text { millions })\end{array}$ & $\begin{array}{l}\triangle \text { QALYs } \\
\text { (thousands) }\end{array}$ & $\begin{array}{l}\text { ICER (if applicable) } \\
\text { [€/QALY] }\end{array}$ \\
\hline \multicolumn{6}{|l|}{ Societal perspective } \\
\hline No vaccination & 2828 & 159 & & & \\
\hline TIV & 3066 & 112 & & & Dominated \\
\hline QIV & 2795 & 97 & & & Dominated \\
\hline TIV/Q-LAIV $100 \%$ & 2717 & 94 & & & Dominated \\
\hline QIV/Q-LAIV $50 \%$ & 2692 & 91 & & & Dominated \\
\hline QIV/Q-LAIV $100 \%$ & 2598 & 86 & -229 & 73 & Cost-saving \\
\hline \multicolumn{6}{|l|}{ Payer perspective } \\
\hline No vaccination & 230 & 159 & & & \\
\hline TIV & 580 & 112 & & & Dominated \\
\hline QIV & 556 & 97 & 327 & 62.4 & 5237 \\
\hline TIV/Q-LAIV $100 \%$ & 628 & 94 & & & Dominated \\
\hline QIV/Q-LAIV $50 \%$ & 586 & 91 & 30 & 5.5 & 5329 \\
\hline QIV/Q-LAIV $100 \%$ & 616 & 86 & 30 & 5.2 & 5734 \\
\hline
\end{tabular}

Scenario names are defined in Table 3. Please see Table S2.2 in the electronic supplementary results for a breakdown of the costs and QALYs $T I V$ trivalent inactivated vaccine, $Q I V$ quadrivalent inactivated vaccine, $Q-L A I V$ quadrivalent live-attenuated influenza vaccine, $Q A L Y$ qualityadjusted life-year, ICER incremental cost-effectiveness ratio, $\triangle$ Costs, $\triangle Q A L Y S$ incremental versus preceding non-dominated scenario

consultations (2800-8800), 170 hospitalisations (70-345), and 18 deaths (6-38) (Table 4).

Switching from TIV to TIV/Q-LAIV $100 \%$ is expected to annually avert, on average, 55,000 symptomatic infections (95\% range 29,000-92,000), 8500 GP consultations (5000-13,500), 300 hospitalisations (150-550) and 35 deaths (17-65).

\subsection{Economic Outcomes}

The 20-year cumulative discounted total costs and QALY losses, calculated for each scenario as the expectation over all simulation runs, are summarised in Table 5. From a societal perspective, from QIV downwards, each successive scenario in Table 5 has lower total costs and fewer QALYs lost than the preceding scenario; each scenario dominates the previous, with the QIV/Q-LAIV $100 \%$ scenario dominating all. All scenarios were expected to be cost saving from a societal perspective compared with no vaccination (Table 5), with the exception of the purely TIV scenario (status quo) which, although not cost saving, is nevertheless likely to be cost effective.

From a payer perspective, the TIV and TIV/Q-LAIV $100 \%$ scenarios were still dominated (Table 5). The remaining scenarios are all likely to be considered cost effective compared with no vaccination, and, in particular, 


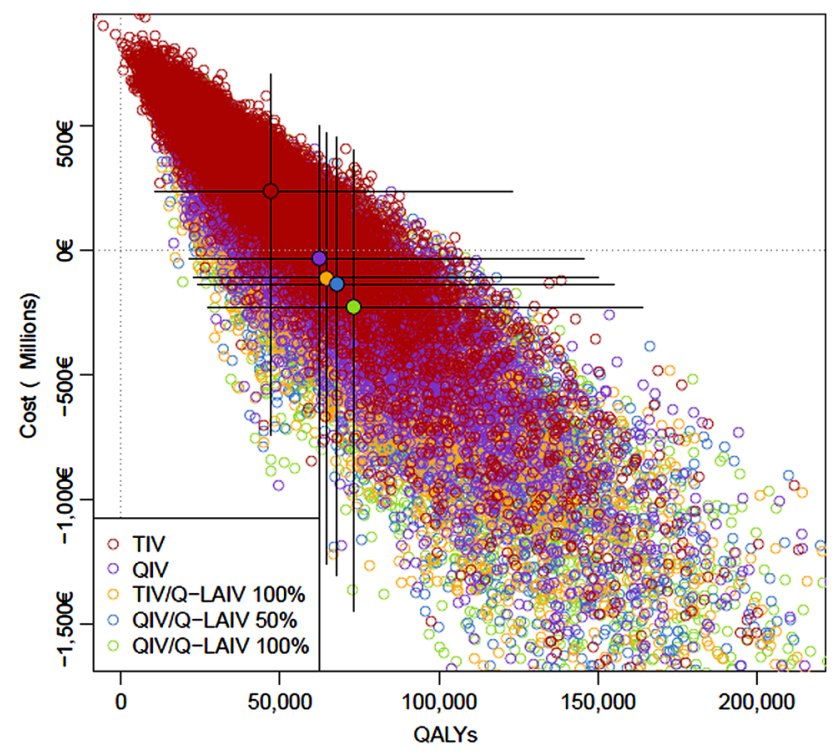

Fig. 5 Discounted incremental costs (2014 Euros) and QALYS of five vaccination scenarios compared with the no vaccination scenario. Bars represent the range in which $95 \%$ of simulations fell. Scenario names in the key are defined in Table 3. QALYs quality-adjusted lifeyears, $T I V$ trivalent inactivated vaccine, $Q I V$ quadrivalent inactivated vaccine, $Q-L A I V$ quadrivalent live-attenuated influenza vaccine

the QIV/Q-LAIV $100 \%$ scenario was the most cost effective in the base case.

On average, the 20-year cumulative discounted saving of switching from the purely TIV scenario to the QIV/QLAIV $100 \%$ scenario was estimated to be $€ 468$ million (including productivity costs) and 26,000 QALYs. The uncertainty around these estimates is considerable (95\% range of simulations $€ 206$ million- $€ 890$ million and 11,100-53,900 QALYS); however, the trend in the expected (mean) values is clear (Fig. 5).

From a societal perspective, the scenario in which Q-LAIV is used exclusively in 2- to $<18$-year-olds had the highest probability of being the most cost-effective scenario, and yielded the highest ENHB at any cost-effectiveness threshold (Fig. 6).

Productivity costs represented the majority of the cost burden of influenza (see Table S2.2 in the electronic supplementary results). When productivity costs were excluded from the model, the scenario in which Q-LAIV had $100 \%$ market share in 2- to $<18$-year-olds had the highest ENHB at thresholds above $€ 6000 / Q A L Y$, and also had the highest probability of being cost effective at thresholds above €6600/QALY (Fig. 6).

\subsection{Sensitivity Analyses}

In the univariate sensitivity analysis, those parameters having a direct effect on the basic reproductive number (transmission coefficient, infectious period) had the greatest influence on the cost effectiveness of individual scenarios. Of those factors that can be most easily changed in practice, dosage price had the greatest influence on costs and QALYs. Relatively large dosage price increases, dependent on the threshold chosen, were possible before the QIV/Q-LAIV $100 \%$ scenario became less preferable in a pairwise comparison with QIV; the same was true of QIV compared with TIV. In all dosage price assumption sensitivity analyses, from either perspective the QIV/Q-LAIV $100 \%$ scenario remained the policy with the highest expected net benefit at cost-effectiveness thresholds above $€ 15,000 / \mathrm{QALY}$. Use of frequency-dependent mixing did not qualitatively change the cost effectiveness of the scenarios. See the electronic supplementary results for more detail.

\section{Discussion}

This study assessed the net health gains within the Finland population of a policy decision to switch from using trivalent to quadrivalent seasonal influenza vaccines. Such a switch is estimated to reduce the incidence of influenzarelated disease, prevent deaths and save healthcare resources.

On average, a switch from trivalent inactivated to QIVs was estimated to result in an $11 \%$ reduction in the average annual number of symptomatic infections, leading to a corresponding 13-16\% reduction in expected cases of medically attended influenza. Influenza-related deaths were estimated to fall by approximately $18 \%$ (an average of 54 lives saved per year).

The use of the live attenuated vaccine in children, with its higher efficacy, nearly doubled the average annual number of symptomatic infections and GP consultations averted by switching from TIV to quadrivalent vaccine, while saving a further 47 and $33 \%$ of influenza-related hospitalisations and deaths, respectively. As most hospitalisations and deaths are in the elderly, a group not indicated for vaccination with Q-LAIV, the majority of these latter savings arise as a result of herd protection.

Considerable uncertainty exists in quantifying many of the processes involved in estimating the epidemiological, clinical and economic impact of switching from trivalent to quadrivalent influenza vaccination.

This study applied a full PSA encompassing the uncertainty in both the transmission dynamics of influenza and the clinical and economic inputs to a model of influenza vaccination. As such, it takes a more thorough account of parameter uncertainty than previous studies of the potential impact of a change in influenza vaccination practice [11, 12, 21, 37-39]. 


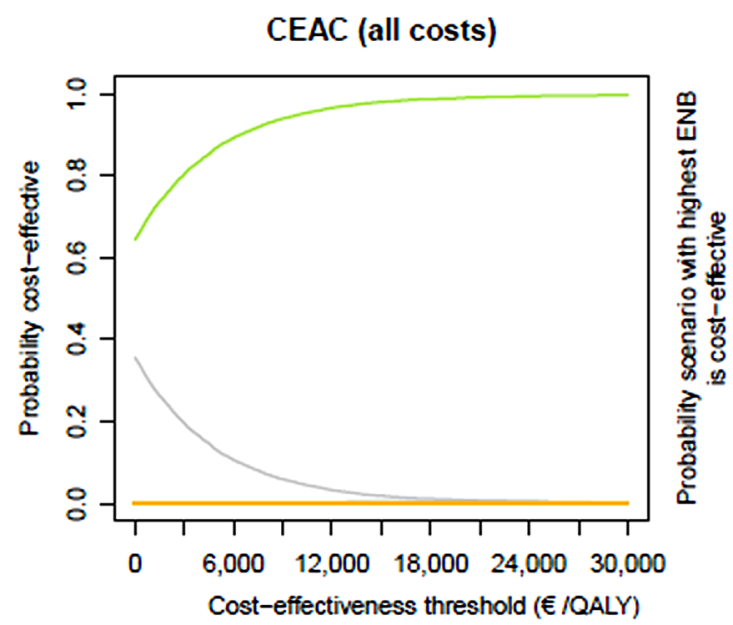

CEAC (productivity costs excluded)

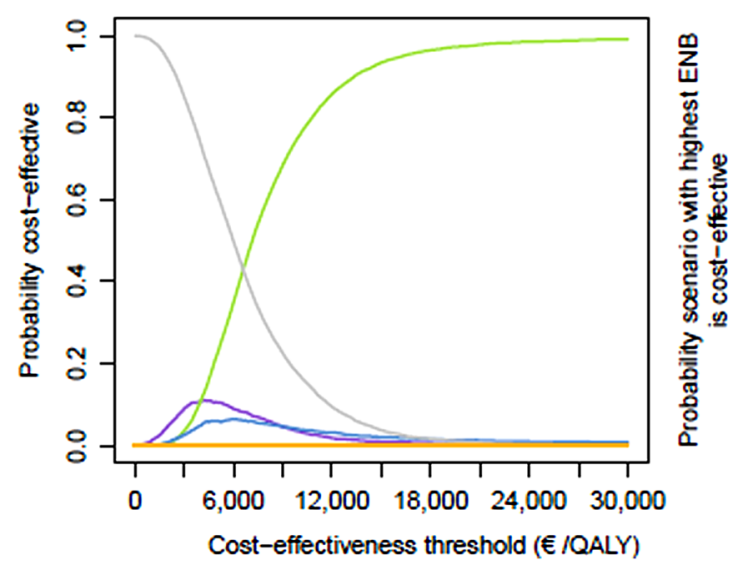

Fig. 6 CEACs and CEAFs for the scenarios presented in Sect. 2.3.1, both including all calculated costs and with productivity costs excluded. Scenario names in the key are defined in Table 3. ENB expected net benefit. CEAC cost-effectiveness acceptability curve,

The PSA, with its method of sampling the transmission parameter distributions and accepting those that fit to the observed paediatric incidence, led to a group of 8998 parameter sets that together form the calibrated model. In the high-dimensional parameter space of this transmission model, there is a danger that fitting routines may be stuck in a local minimum, and no guarantee that any minimum found corresponds to the real-world situation; these limitations are avoided by our method, which samples across parameter space. While a parameter set exists that provides the best fit (lowest deviance) compared with the data [22] calibrated over the 2000-2002 influenza seasons, there is no specific parameter set that will yield the average model results, or yield best-fitting results over an extended time period. However, as shown in Fig. 2, the aggregate model
CEAF (all costs)

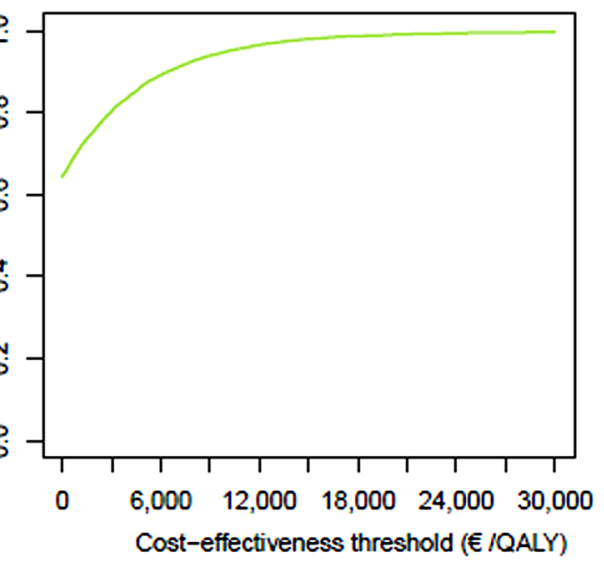

CEAF (productivity costs excluded)

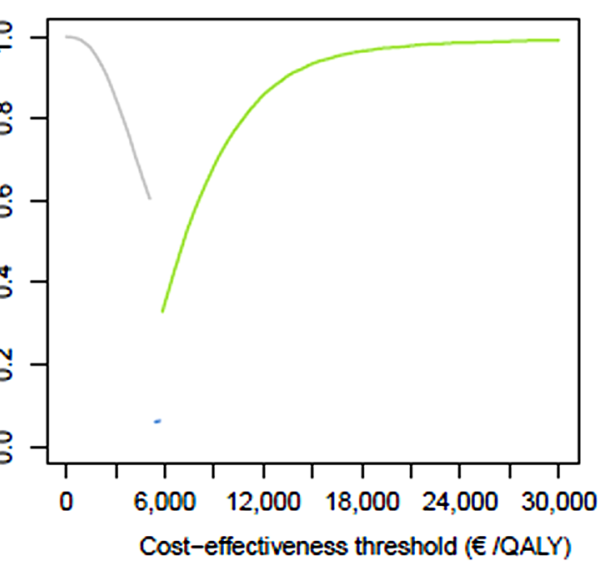

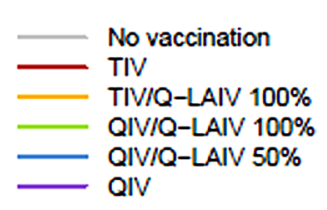

$C E A F$ cost-effectiveness acceptability frontier, $Q A L Y$ quality-adjusted life-year, $T I V$ trivalent inactivated vaccine, $Q I V$ quadrivalent inactivated vaccine, $Q-L A I V$ quadrivalent live-attenuated influenza vaccine

behaviour closely encompasses the observed Finnish incidence of influenza.

As is evident from the univariate sensitivity analysis, the most influential parameters are those that are key drivers of the transmission dynamics. Despite the uncertainty surrounding the magnitude and shape of the input distributions for the parameters that determine $R_{0}$, clear unimodal parameter distributions were obtained for the basic reproductive number, with mean values for each subtype and lineage falling between 1.5 and 2.2, consistent with previous estimates [40, 41].

Vaccination was implemented in a pulse prior to the start of the season. This was a limitation as it did not take account of the ramp-up time required to reach a particular level of coverage, and therefore over estimated the person days spent protected in any one season. Univariate analyses 
showed the uptake level had a moderate effect on the model outcomes.

The survey of vaccine uptake, conducted as part of this study, revealed that vaccine coverage in children over 36 months, not currently recommended for vaccination, was comparable with and possibly higher (22-28\%) than in children under 4 years of age $(23 \%)$, reflecting the mixed private/public nature of healthcare in Finland. These higher rates are possibly because individuals with relatives in an at-risk group are also indicated for free vaccination [42], and therefore may have been vaccinated for the sake of those relatives. Despite this observation, paediatric coverage is below that seen in some other countries, notably the US, where vaccine uptake in children has exceeded $50 \%$ since 2010 [43]. If vaccine uptake in children were to increase, then it is likely that even greater reductions in influenza-related disease could be achieved. This is particularly true for policies involving the use of quadrivalent vaccines, where the effect of higher uptake is not diluted by periodic mismatching between the influenza B lineage used for the vaccine and that circulating in the population.

A lack of seroepidemiological data from Finland means that it is not possible to directly validate the model's simulated incidence of infection, independent of symptomatic presentation. The probability of symptoms, given an influenza infection, are however based on a meta-analysis of challenge studies [44]. Although these challenge studies were conducted exclusively in young adults, the subsequent correspondence achieved in the temporal pattern of average annual symptomatic disease incidence between the model and surveillance data published by THL is reassuring, particularly as the THL data were not used in the calibration process and the simulated incidence of symptomatic infection is highly sensitive to the probability of an infection being symptomatic.

No previous studies exist of the cost effectiveness of introducing quadrivalent influenza vaccination in Finland; however, published studies exist from elsewhere, including the US [11, 14], Canada [15] and the UK [15, 37]. In contrast with the dynamic model used in this paper, two of these studies [11,37] use static models and will therefore underestimate the number of clinical events averted by vaccination and hence undervalue quadrivalent vaccination. All three studies estimate quadrivalent vaccination to be cost effective. Differences in population size, density and pre-existing immunity due to historical influenza transmission and vaccination coverage mean that the underlying transmission dynamics of influenza are likely to differ between these countries. This, together with different model structures, makes it difficult to directly compare estimates of averted costs and QALYs across studies.
While parameter uncertainty has been thoroughly explored in a PSA on the transmission as well as clinical and economic parameters, this has been undertaken within the constraints imposed by the structure of the model. The structural assumptions made by the current dynamic transmission model have been previously published $[16,21,38]$ and are outlined in detail in the electronic supplementary material. One of these assumptions is a lack of cross-protection between different lineages of influenza B. Recent analyses have suggested that some degree of cross-protection may exist [36, 45, 46], the consequence of which would be to reduce the incremental benefit of quadrivalent vaccines over their trivalent counterparts. The magnitude of this cross-protection remains unclear, with estimates ranging from 0 to $60 \%$.

\section{Conclusions}

This analysis demonstrates that quadrivalent vaccination is likely to be highly cost effective. From a societal perspective, of those scenarios examined, the use of Q-LAIV in children aged 2 to $<18$ years of age, and QIV in all other age groups, had the highest probability of being cost effective at all costeffectiveness thresholds, and yielded the highest ENHB. If productivity costs are excluded, from a payer perspective this scenario has the highest ENHB at cost-effectiveness thresholds above $€ 6000 / \mathrm{QALY}$. A switch to such a policy is likely to be highly cost effective, reducing the burden of influenzarelated disease and freeing up healthcare resources.

Acknowledgments The authors would like to thank TNS Sifo/TNS Gallup Oy for carrying out the vaccine uptake survey. Lisa Nagy and Richard Pitman coded, calibrated and ran the model, drafted the manuscript, and approved the final version. Alfred Sackeyfio assisted with interpretation of the results, critically revised the manuscript, and approved the final version. Terho Heikkinen provided conceptual guidance, provided the data used for calibration, critically revised the manuscript, and approved the final version.

\section{Compliance with Ethical standards}

Conflict of interest This study was sponsored by AstraZeneca, of which Alfred Sackeyfio is an employee. The work and opinions presented in this manuscript are solely those of the authors. Richard Pitman has participated as a member of AstraZeneca advisory boards and received funding for research projects. Terho Heikkinen has given a lecture at an academic symposium organized by AstraZeneca but was not compensated for his work on this article.

Open Access This article is distributed under the terms of the Creative Commons Attribution-NonCommercial 4.0 International License (http://creativecommons.org/licenses/by-nc/4.0/), which permits any noncommercial use, distribution, and reproduction in any medium, provided you give appropriate credit to the original author(s) and the source, provide a link to the Creative Commons license, and indicate if changes were made. 


\section{References}

1. Muennig PA, Khan K. Cost-effectiveness of vaccination versus treatment of influenza in healthy adolescents and adults. Clin Infect Dis. 2001;33(11):1879-85.

2. Luce BR, Zangwill KM, Palmer CS, Mendelman PM, Yan L, Wolff MC, et al. Cost-effectiveness analysis of an intranasal influenza vaccine for the prevention of influenza in healthy children. Pediatrics. 2001;108(2):E24.

3. Salo H, Kilpi T, Sintonen H, Linna M, Peltola V, Heikkinen T. Cost-effectiveness of influenza vaccination of healthy children. Vaccine. 2006;24(23):4934-41.

4. Belshe RB. The need for quadrivalent vaccine against seasonal influenza. Vaccine. 2010;28(Suppl 4):D45-53.

5. Eichner M, Schwehm M, Hain J, Uphoff H, Salzberger B, Knuf $\mathrm{M}$, et al. 4Flu —an individual based simulation tool to study the effects of quadrivalent vaccination on seasonal influenza in Germany. BMC Infect Dis. 2014;14(1):365.

6. Mereckiene J, Cotter S, D’Ancona F, Giambi C, Nicoll A, LévyBruhl D, et al. Differences in national influenza vaccination policies across the European Union, Norway and Iceland 2008-2009. Euro Surveill. 2010;15(44):1-10 (pii = 19700). http://www.eurosurveillance.org/ViewArticle.aspx?ArticleId= 19700

7. Anundi E, Holmstrom P, Kela E, editors. Infectious diseases in Finland 2002. National Public Health Institute (KTL); 2003. p. 10.

8. Jaakola S, Lyytikäinen O, Rimhanen-Finne R, Salmenlinna S, Vuopio J, Roivainen M, et al. Infectious diseases in Finland 2011. National Institute for Health and Welfare (THL); 2012. p. 38.

9. Heikkinen T, Ikonen N, Ziegler T. Impact of influenza B lineagelevel mismatch between trivalent seasonal influenza vaccines and circulating viruses, 1999-2012. Clin Infect Dis. 2014;59(11):1519-24.

10. Ambrose CS, Levin MJ. The rationale for quadrivalent influenza vaccines. Hum Vaccin Immunother. 2012;8(1):81-8.

11. Clements K, McGarry L, Pruttivarasin N, Misurski DA, Meier G. Cost-effectiveness analysis of universal influenza vaccination with quadrivalent inactivated vaccine in the United States. Hum Vaccin Immunother. 2014;10(5):1171-80.

12. Lee B, Bartsch S, Willig A. The economic value of a quadrivalent versus trivalent influenza vaccine. Vaccine. 2012;30(52):7443-6.

13. Pitman R, Fisman D, Zaric GS, Postma M, Kretzschmar M, Edmunds J, et al. Dynamic transmission modeling: a report of the ISPOR-SMDM Modeling Good Research Practices Task Force Working Group-5. Med Decis Mak. 2012;32:712-21.

14. Crépey P, Boer PT, Postma MJ, Pitman R. Retrospective public health impact of a quadrivalent influenza vaccine in the United States. Influenza Other Respir Viruses. 2015;9(Suppl 1):39-46.

15. Thommes EW, Ismaila A, Chit A, Meier G, Bauch CT. Costeffectiveness evaluation of quadrivalent influenza vaccines for seasonal influenza prevention: a dynamic modeling study of Canada and the United Kingdom. BMC Infect Dis. 2015;15:465-78.

16. Vynnycky E, Pitman R, Siddiqui R, Gay N, Edmunds WJ. Estimating the impact of childhood influenza vaccination programmes in England and Wales. Vaccine. 2008;26(41):5321-30.

17. Official Statistics of Finland (OSF). Population structure [epublication]. Helsinki. Available at: http://www.stat.fi/til/vaerak/ tau_en.html. Accessed 26 Nov 2013.

18. Official Statistics of Finland (OSF). Deaths [e-publication]. Helsinki. Available at: http://www.stat.fi/til/kuol/index_en. html. Accessed 26 Nov 2013.
19. Mossong J, Hens N, Jit M, Beutels P, Auranen K, Mikolajczyk R, et al. Social contacts and mixing patterns relevant to the spread of infectious diseases. PLoS Med. 2008;5(3):381-91.

20. World Health Organization (WHO). Influenza vaccine viruses and reagents. Available at: http://www.who.int/influenza/ vaccines/virus/en/. Accessed 18 May 2014.

21. Pitman RJ, Nagy LD, Sculpher MJ. Cost-effectiveness of childhood influenza vaccination in England and Wales: results from a dynamic transmission model. Vaccine. 2013;31:927-42.

22. Heikkinen T, Silvennoinen H, Peltola V, Ziegler T, Vainionpaa $\mathrm{R}$, Vuorinen $\mathrm{T}$, et al. Burden of influenza in children in the community. J Infect Dis. 2004;190(8):1369-73.

23. World Health Organization (WHO). WHO FluNet database. Available at: http://www.who.int/influenza/gisrs_laboratory/ flunet/en/. Accessed 2 May 2014.

24. Silvennoinen $H$, Peltola $V$, Vainionpää $R$, Ruuskanen $O$, Heikkinen T. Incidence of influenza-related hospitalizations in different age groups of children in Finland: a 16-year study. Pediatr Infect Dis J. 2011;30:e24-8.

25. Official Statistics of Finland (OSF). Causes of death [e-publication]. Helsink. Available at: http://www.stat.fi/til/ksyyt/index_en. html. Accessed 18 May 2014.

26. Pitman R, Nagy L, Scott D. Cost-effectiveness of quadrivalent influenza vaccination in England and Wales. In: Options for the control of influenza VIII, Cape Town, South Africa [poster P1-195]; 2013.

27. Pharmaceuticals Pricing Board. Application instructions for a health economic evaluation; 2013. Available at: http://www.stm. fi/en/ministry/boards/pharmaboard/application_forms. Accessed 4 Feb 2013.

28. Kapiainen S, Vaisanen A, Haula T. Terveyden- ja sosiaalihuollony ksikkökustannukset Suomessavuonna 2011 (Health and social care unit costs in Finland 2011). National Institute for Health and Welfare (THL); 2014. Available at: https://www. julkari.fi/bitstream/handle/10024/114683/THL_RAPO3_2014_ web.pdf?sequence=1. Accessed 6 Aug 2014 .

29. Kela medicinal products database. Social Insurance Institution of Finland (Kela). Available at: https://easiointi.kela.fi/laakekys_ app/LaakekysApplication?kieli=en. Accessed 16 May 2014.

30. Official Statistics of Finland (OSF). Consumer price index [epublication]. Helsinki. Available at: http://www.stat.fi/til/khi/ index_en.html. Accessed 18 May 2014.

31. Official Statistics of Finland (OSF). Wage and salary indices [epublication]. Helsinki. Available: at: http://www.stat.fi/til/ktps/ index_en.html. Accessed 18 May 2014.

32. Application instructions: Health economic evaluation. Lääkkeiden Hintalautakunta Läkemedelsprisnämnden (Pharmaceuticals Pricing Board); 2011.

33. Turner D, Wailoo A, Nicholson K, Cooper N, Sutton A, Abrams K. Systematic review and economic decision modelling for the prevention and treatment of influenza A and B. Health Technol Assess. 2003;7(35):iii-iv, xi-xiii, 1-170.

34. Ara R, Brazier JE. Populating an economic model with health state utility values: moving toward better practice. Value Health. 2010;13(5):509-18.

35. Fenwick E, Claxton K, Sculpher M. Representing uncertainty: the role of cost-effectiveness acceptability curves. Health Econ. 2001;10(8):779-87.

36. DiazGranados CA, Denis M, Plotkin S. Seasonal influenza vaccine efficacy and its determinants in children and non-elderly adults: a systematic review with meta-analyses of controlled trials. Vaccine. 2012;31:49-57.

37. Van Bellinghen LA, Meier G, Van Vlaenderen I. The potential cost-effectiveness of quadrivalent versus trivalent influenza vaccine in elderly people and clinical risk groups in the uk: a lifetime multi-cohort model. PLoS One. 2014;9(6):e98437. 
38. Pitman R, White L, Sculpher M. Estimating the clinical impact of introducing paediatric influenza vaccination in England and Wales. Vaccine. 2012;30:1208-24.

39. Reed C, Meltzer MI, Finelli L, Fiore A. Public health impact of including two lineages of influenza $\mathrm{B}$ in a quadrivalent seasonal influenza vaccine. Vaccine. 2012;30(11):1993-8.

40. Longini IM, Halloran ME, Nizam A, Yang Y. Containing pandemic influenza with antiviral agents. Am J Epidemiol. 2004;159(7):623-33.

41. Vynnycky E, Edmunds WJ. Analyses of the 1957 (Asian) influenza pandemic in the United Kingdom and the impact of school closures. Epidemiol Infect. 2008;136(2):166-79.

42. Vakavalle influenss allealttiiden lähipiirin influenssarokotukset; 2014 [Finnish]. Available at: http://www.thl.fi/fi/web/ rokottaminen/rokotteet/kausi-influenssarokote/vakavalleinfluenssalle-alttiiden-lahipiiri. Accessed 26 Feb 2015.

43. Centers for Disease Control and Prevention (CDC). Influenza vaccine coverage. Available at http://www.cdc.gov/flu/ fluvaxview/coverage-1213estimates.htm\#data. Accessed 24 Sept 2014.

44. Carrat F, Vergu E, Ferguson NM, Lemaitre M, Cauchemez S, Leach S, et al. Time lines of infection and disease in human influenza: a review of volunteer challenge studies. Am J Epidemiol. 2008;167(7):775-85.

45. Belshe RB, Coelingh K, Ambrose CS, Woo JC, Wu X. Efficacy of live attenuated influenza vaccine in children against influenza $B$ viruses by lineage and antigenic similarity. Vaccine. 2010;28(9):2149-56.

46. Osterholm MT, Kelley NS, Sommer A, Belongia EA. Efficacy and effectiveness of influenza vaccines: a systematic review and meta-analysis. Lancet Infect Dis. 2012;12(1):36-44.

47. Rivetti D, Jefferson T, Thomas R, Rudin M, Rivetti A, Pietrantonj $\mathrm{CD}$, et al. Vaccines for preventing influenza in the elderly. Cochrane Database Syst Rev. 2006;(3):CD004876. 